\title{
La collection éclatée
}

Circulations et techniques d'organisation des données en sciences naturelles (XVIII ${ }^{\mathrm{e}}$-début XIX ${ }^{\mathrm{e}}$ siècle)

The broken collection. Circulations and techniques for organizing data in natural sciences ( $18^{\text {th }}$ century-beginning of the $19^{\text {th }}$ century)

\section{Thérèse Bru}

\section{(2) OpenEdition}

\section{Journals}

Édition électronique

URL : http://journals.openedition.org/artefact/872

DOI : $10.4000 /$ artefact. 872

ISSN : 2606-9245

Éditeur :

Association Artefact. Techniques histoire et sciences humaines, Presses universitaires du Midi

Édition imprimée

Pagination : 157-176

ISBN : 978-2-7535-7305-5

ISSN : 2273-0753

Référence électronique

Thérèse Bru, «La collection éclatée », Artefact [En ligne], 6 | 2017, mis en ligne le 31 mai 2018, consulté le 06 mars 2020. URL : http://journals.openedition.org/artefact/872 ; DOI : https://doi.org/10.4000/ artefact. 872 


\section{La collection éclatée. Circulations et techniques d'organisation des données en sciences naturelles (XVIII ${ }^{\mathrm{e}}$-début $\mathrm{XIX} \mathrm{X}^{\mathrm{e}}$ siècle)}

Thérèse BRU*

\section{Résumé}

Les biologistes du $\mathrm{XVIII}^{\mathrm{e}}$ siècle n'ont pas seulement fait face à un accroissement exponentiel du nombre des espèces découvertes à travers le globe, notamment à partir des circumnavigations des années 1760 . Ils ont été aussi confrontés au problème de la dispersion et de la recomposition incessante des collections de spécimens et de matériaux de travail. L'éclatement des collections, aux mains d'une multitude d'acteurs privés comme d'institutions nationales et de sociétés scientifiques, représente un défi pour l'établissement de classifications et de taxonomies fiables sur lesquels est construite toute l'architecture technique d'analyse du vivant. Une part importante des correspondances en sciences naturelles confronte ainsi des données venant de collections personnelles éclatées et vise à éviter l'émergence de doublons dans la littérature scientifique. La collection suit la forme du graphe et en a l'étendue : c'est la notion même de collection qui est recomposée au prisme de la circulation des données, tandis que les réseaux d'échange scientifiques deviennent un dispositif technique essentiel à l'exploitation des stocks de matériaux et d'échantillons disponibles.

*. Normalienne, agrégée d'histoire, doctorante au sein de l'UMR 8533 IDHES de l'université Paris 8, Thérèse Bru consacre sa thèse aux naturalistes et à leurs données entre les mondes britannique et français (1700-1836). Dernières publications : Thérèse BRU, « Plus vrai que nature. Conversion des objets en information scientifique, conversion de l'information en objets dans les correspondances en sciences naturelles ", in Thérèse Bru et Solène de LA Forest D'ARMAILlé, Matière à écrire. Les échanges de correspondance du XVI $I^{e}$ au XIX siècle, Vincennes, Presses universitaires de Vincennes, coll. Temps et espaces, 2017, p. 179-203; id., « Science à distance. Cultures expérimentales, transferts de données et mutations des pratiques scientifiques dans les mondes britannique et français, XVIII ${ }^{\mathrm{e}}-\mathrm{XIX}^{\mathrm{e}}$ siècles ", in Thomas PRÉvERAUD (dir.), Circulations savantes entre l'Europe et le monde, XVIt $-\mathrm{XX}$ siècle, PUR, coll. Enquêtes et documents, 2017. Contact : [therese.bru@gmail.com]. 
Mots clefs : collections scientifiques, données massives, histoire de l'information, musées, réseaux, sciences naturelles XVIII-XIXe siècles, spécimens scientifiques, techniques de collection.

\section{Abstract : The broken collection. Circulations and techniques for organizing data in natural sciences $\left(18^{\text {th }}\right.$ century - beginning of the $19^{\text {th }}$ century)}

During the $18^{\text {th }}$ century, biologists were not only confronted with the exponential growth in the number of species discovered around the world, especially since the circumnavigations of the 1760s. They also faced the problem of dispersion and incessant recomposition of collections of specimens and work materials. Fragmented collections and informations, in the hands of a multitude of private actors as well as of national institutions and a vast array of scientific societies, represented a challenge while the need for reliable classifications and taxonomies was affecting the burgeoning understanding of order in nature. Most of the remaining correspondences show how time was spent dealing with unresolved names, comparing specimens and data preserved in hundreds of personal collections in order to prevent the spread of duplicates in the scientific literature. The graph, in itself, was the extent of the collection, whose very notion was recomposed through the prism of information circulation, while scientific exchange networks became a technical device essential to the analysis of increasingly massive samples and data sets.

Keywords : big data, collecting practices, history of information, museums, natural sciences (18 $8^{\text {th }}-19^{\text {th }}$ centuries), networks, scientific collections, scientific specimens.

La collection est généralement associée au musée, dont elle constitue la raison d'être et bien souvent l'acte de naissance. Le caractère cumulatif des collections modernes, alimenté par de multiples dons, a tendance à faire oublier les origines souvent individuelles $\mathrm{du}$ processus de collecte formant le noyau originel du dépôt. Le British Museum a pour embryon les collections du naturaliste Hans Sloane (1660-1753), véritable réduction du monde en son temps. Le Conservatoire des arts et métiers repose sur le noyau originel d'un legs au roi de machines par Jacques Vaucanson (1709-1782). Les processus de collecte et d'accumulation de matériaux scientifiques et techniques commencent entre des mains individuelles, à une échelle infra-institutionnelle et infra-étatique.

Ce sont les chemins de traverse de l'accumulation individuelle qu'emprunte cette contribution, la masse obscure des échantillons personnels qu'elle examine. Avant l'avènement des dépôts institutionnels centralisés (les muséums du XIX ${ }^{\mathrm{e}}$ siècle), au XVIII ${ }^{\mathrm{e}}$ siècle, une importante quantité de naturalistes 
acquièrent pour leur propre compte des spécimens botaniques, zoologiques ou minéralogiques. Cette dynamique massive mais éclatée de collecte est accentuée par une caractéristique propre aux matériaux en sciences naturelles : un seul spécimen peut modifier une théorie ${ }^{1}$. Mille échantillons ne suffisent pas à la confirmer, mais un seul est suffisant pour la détruire. La recherche du hapax rend dès lors nécessaire l'examen de grandes masses de données. Par « données ", nous entendrons, dans cet article, l'ensemble des objets matériels (spécimens, échantillons, etc.) et immatériels (mesures, paramètres) extraits du monde physique qui constituent la matière première des travaux des naturalistes, leurs matériaux de travail ${ }^{2}$. Accumuler le plus de matériaux possible participe de l'obsession $\mathrm{du} \mathrm{XVIII}^{\mathrm{e}}$ siècle pour la création de systèmes décrivant les espèces et de sa hantise de voir se briser ces architectures sur un seul spécimen.

Cette extension de la collecte a généré sur le long xvIII $^{\mathrm{e}}$ siècle des collections éclatées entre une multitude de mains particulières. Fragmentées et fragmentaires, elles ont souvent été étudiées au cas par cas par nombre de monographies centrées sur la personne d'un naturaliste. Ces collections éclatées ont rarement été envisagées comme un tout.

L'objet de cet article est ce qui relie ces collections : leur connexion et intersection, envisagées en tant qu'espace de travail pour les naturalistes. Nous proposons d'observer ce qui se passe dans les interstices de ces masses variables de spécimens accumulés selon des logiques propres, mais reliés par un réseau de mentions, de prêts et d'emprunts. En somme, nous proposons d'étudier la for- mation de collections virtuelles, constituées de plusieurs dépôts qui peuvent être mis en relation à tout moment par la comparaison des matériaux. Ce faisant, nous introduisons une idée de collection qui dépasse le simple regroupement d'objets dans un même lieu. Des processus d'échange suffisamment nombreux entre des stocks de matériaux scientifiques dispersés accréditent l'idée que ce qui se passe entre les collections participe de la collection elle-même. Si, dans la seconde moitié du $\mathrm{XVIII}^{\mathrm{e}}$ siècle, les institutions centrales organisent de plus en plus la collecte du monde ${ }^{3}$, elles ont néanmoins à gérer la multiplication croissante des stocks privés d'informations et de matériaux qui représentent un gisement bien plus important que tout ce qu'elles peuvent réunir, même à un échelon national. C'est ainsi que l'historien peut gagner à s'écarter du centre des collections pour porter son attention à l'intersection de celles-ci, cette intersection que crée une circulation accrue des matériaux depuis le $\mathrm{xvIII}^{\mathrm{e}}$ siècle. $\mathrm{Ce}$ changement d'échelle constitue un réétalonnage de la portée des collections, vers un niveau de méta-organisation porteur de conséquences sur les techniques de traitement des données dans les sciences. Mais c'est aussi un changement de nature de ce que signifie l'action même de collecter. 


\section{L'invention de la collection scientifique et le tropisme de la centralisation}

La collection en sciences naturelles ne va pas de soi. Ce concept correspondant à des pratiques nouvelles se solidifie progressivement au cours de l'ère moderne, comme le montrent les variations terminologiques autour de ce vocable mal figé. Sarah Irving a relevé qu'Henry Oldenburg et les autres savants formant le noyau originel de la Royal Society de Londres utilisent différents termes pour désigner la collection de la jeune société, plus couramment nommée Repository of the Royal Society ${ }^{4}$ : il n'existe encore aucun terme adéquat dans le vocabulaire de la curiosité et du patronage de cour pour caractériser la dimension systématique de cette entreprise nouvelle. Les termes de "raretés ", "spécimens " apparaissent dans les lettres du début du xvIII ${ }^{\mathrm{e}}$ siècle; celui de "sample " (échantillon) dans les Philosophical transactions. Une analyse terminologique fondée sur les vastes corpus numérisés par Google montre que le terme de " collection" ne décolle, dans l'usage, que vers $1750^{5}$. La hausse des occurrences est ensuite constante jusqu'en 1800, après quoi elle se maintient à un pallier stable jusqu'en 1900.

Le concept de collection est un outil technique en lui-même : l'idée qu'il existe une valeur heuristique dans le rassemblement des objets, une valeur supérieure à la somme de ces objets individuels, reflète déjà à quel point la collection opère comme un ensemble de liens. Durant la première moitié du xviII ${ }^{\mathrm{e}}$ siècle, émerge une nouvelle approche systématique de la nature visible dans l'accéléra- tion des tentatives d'établir des systèmes de classement, approche plus que jamais dépendante d'une infrastructure technique articulant les objets du monde naturel, pensée pour sa réduction et son étude. Au milieu du xvıII ${ }^{e}$ siècle, en effet, les tentatives de classification des espèces se multiplient, dans la lignée des travaux de nomenclature botanique de savants comme le Suédois Carl Linné ou le Français Michel Adanson. Pour dégager un ordre dans la nature, un grand nombre de spécimens nouveaux sont nécessaires afin d'étalonner les espèces connues et les regrouper en embranchements principaux. Cette période coïncide avec le départ de circumnavigations vers l'hémisphère sud et un renouveau de l'exploration, cette fois portée par les appareils étatiques. Dans les années 1770, alors que les résultats des voyages d'exploration arrivent sur le marché de l'édition en même temps que de grandes quantités d'échantillons nouveaux, la volonté scientifique est au rassemblement des données disponibles en systèmes (souvent concurrents) : celui de Linné conquiert la Grande-Bretagne tandis que les institutions françaises sont dominées par celui de Jussieu. Le rassemblement de masses importantes de données est une condition nécessaire à la formation et la rectification de tels systèmes.

C'est dans ce contexte que la collection de travail scientifique émerge comme une force centripète appliquée à un ensemble d'objets, visant à créer un point de rassemblement, une réduction 
du monde : un groupe d'éléments représentatifs et exploitables. En ce sens, la collection constitue une tentative de simplification des ensembles de données ou de corps présents dans le monde naturel, mais aussi des liens qui les unissent.

Alors que cette masse de matériaux nouveaux, extra-européens, afflue sur le marché des échantillons du monde savant, leur agrégation et organisation rentrent également dans les intérêts d'États engagés dans des stratégies d'expansion mondiale. Pour progresser dans le monde, il faut des données aux puissances impériales européennes ${ }^{6}$, au premier rang desquelles les deux principales restées en lice, l'Angleterre et la France. L'organisation de cette matière première venue du monde entier devient un enjeu direct de stratégies impériales qui se détournent de l'Amérique du Nord après 1763 et 1783 . La puissance des institutions scientifiques et des groupes qui les dirigent se construisent sur l'agencement de ces matériaux nouveaux. Ce n'est donc pas un hasard si les occurrences du terme " collection » se multiplient rapidement dans la seconde moitié du xviII ${ }^{e}$ siècle : il existe une corrélation entre la concentration du pouvoir de nommer et d'organiser dans les institutions centrales comme la Royal Society, l'Académie royale des sciences ou le Cabinet du Roi à Paris et l'émergence de la collection. La puissance des institutions et le renom des grands naturalistes " classificateurs", tels que Jussieu, se construit sur la centralisation des objets et des données.

$C^{\prime}$ est ainsi que se consolide un système de concentration des matériaux, la collection, qui va a priori dans le sens opposé à celui de la dispersion. C'est pourtant dans le sillage de la concentration des matériaux que vont se développer les conditions de leur éclatement.

\section{Un double processus d'intégration verticale et de prolifération horizontale}

Deux processus simultanés sont à l'œuvre, dont les effets se renforcent mutuellement.

Le premier est un mouvement d'intégration verticale des collections, observable sur le temps long, et particulièrement visible en ce qui concerne les herbiers botaniques. Par achat, don, legs ou préemption, les collections particulières sont absorbées les unes dans les autres à mesure que disparaissent leurs possesseurs, créant des emboîtements complexes d'ensembles issus de fonds très différents. La nécessité croissante de disposer de matériaux étendus pour se repérer dans les architectures classificatoires naissantes donne l'impulsion à des réunions d'herbiers dont certains, truffés de spécimens rares, font l'objet d'enchères convoitées tant par les spécialistes que par les pouvoirs étatiques.

En témoigne le sort de la collection botanique massive du botaniste Sébastien Vaillant (1669-1722), tributaire d'une intégration d'herbiers acquis auprès d'autres naturalistes (graphe 1). 


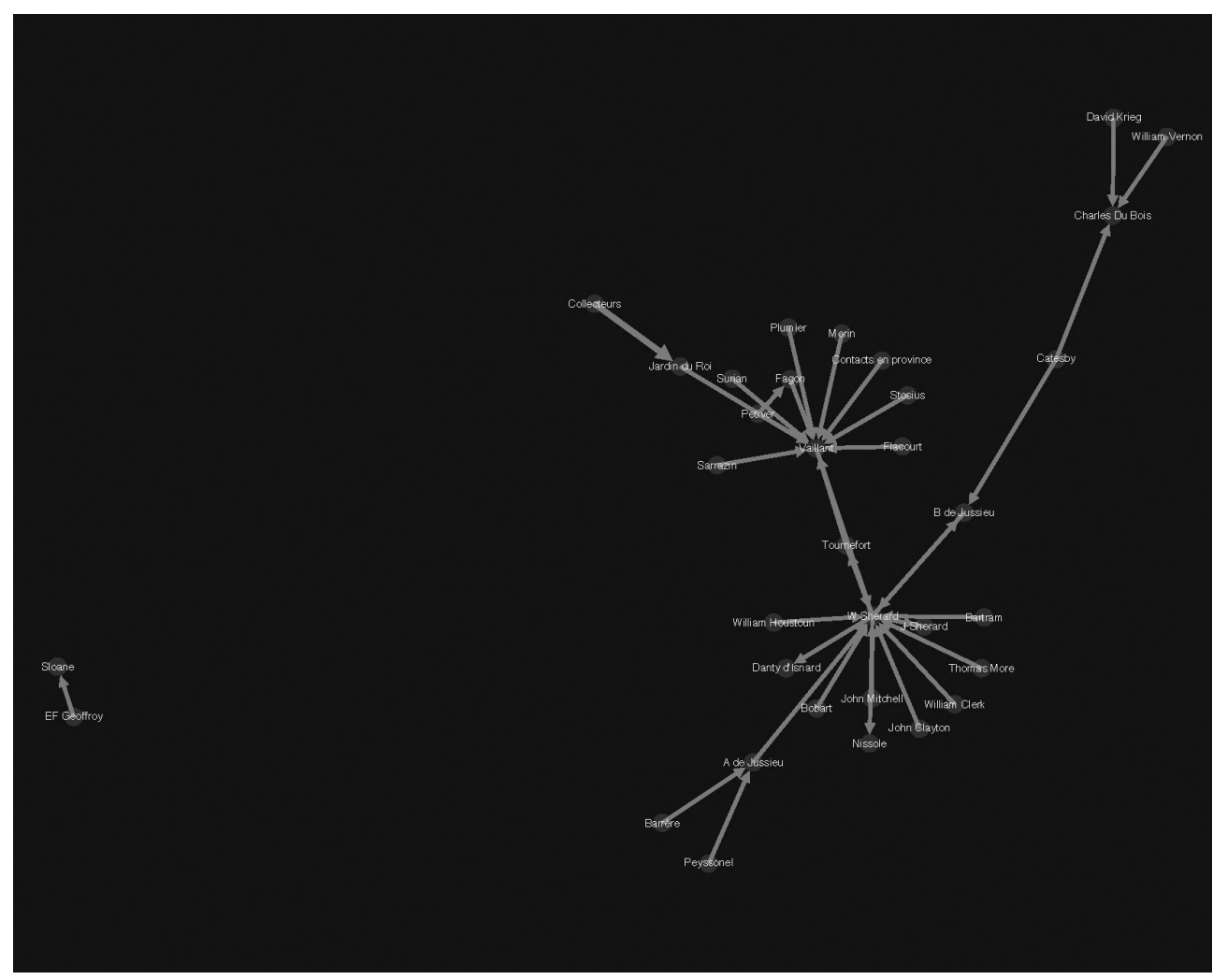

Graphe 1. - Intégration verticale : graphe simplifié des dépendances des collections de Vaillant et Sherard à des stocks antérieurs de matériaux. Les noms des collecteurs les ayant alimentées rendent visibles les différentes chaînes de remontée de l'information.

Le rôle structurant des réseaux étatiques dans les mécanismes de structuration verticale est ici visible dans la très forte intégration du réseau d'informateurs et de fournisseurs de Vaillant dans les institutions royales (Jardin du roi et Académie des sciences) qui contribuent largement à sa surface sociale en termes d'influence et de collecte. Avant de mourir, Vaillant s'efforce de transférer l'une des plus riches collections d'Europe à son ami anglais William Sherard (16581728), lui-même engagé dans des stratégies d'intégration de matériaux pour son propre herbier. En incorporant l'herbier de Vaillant aux collections de Sherard, l'ensemble deviendrait l'un des premiers
d'Europe. Vaillant, par une mise en abîme du processus dans lequel il est luimême engagé, liste les collections que son propre herbier a phagocytées :

«Vous savez Monsieur que [Vaillant] a vu l'herbier de Monsieur Tournefort, qu'il a parcouru celui de Monsieur Morin, que Monsieur Fagon lui a donné le sien. Vous savez que Monsieur Vaillant a desséché toutes les plantes qui ont été cultivées au jardin royal à Paris depuis trente années environ. Vous savez aussi que M[ichel] Sarrazin lui a envoyé toutes celles qu'il a découvertes dans ses courses; que 
le R[évérend] P[ère] Plumier lui en a donné plusieurs; que monsieur Tournefort lui a donné celles qu'il a découvertes dans ses voyages, qu'il a eu des correspondances dans une partie de provinces de France, qu'il a fait lui-même quelques voyages dans lesquels il a ramassé beaucoup de plantes, que ces mêmes correspondances se sont étendues jusque dans les pays étrangers ${ }^{7}$. »

L'intégration verticale est clairement visible dans ces ensembles emboîtés comme des poupées gigognes qui sont sur le point de rejoindre les collections de Sherard. Mais, au dernier moment, le roi de France court-circuite la passation des échantillons et préempte la collection qui est intégrée au Cabinet du Roi - sans doute sur les bons conseils de Jussieu, ennemi de Vaillant et peu désireux de voir les précieuses collections quitter le pays. Sherard doit s'incliner devant l'offre royale et le processus d'intégration rejoint le noyau de ce qui deviendra l'herbier du Muséum d'histoire naturelle ${ }^{8}$. Le sort des collections de Vaillant, qu'une amitié transnationale de longue date ne parvient pas à soustraire à la capacité d'attraction de l'État, montre que ce processus d'intégration vertical des collections est de plus en plus corrélé avec la puissance montante des institutions d'État. Les achats successifs de sous-ensembles agglomérés à des stocks préexistants sont en effet coûteux et, seuls, quelques particuliers fortunés peuvent rivaliser dans ces enchères avec la capacité d'attraction de l'État. Ces particuliers se comptent sur les doigts d'une main et sont souvent britanniques, à l'instar de Joseph Banks (1743-1820), devenu une personnalité publique et un conseiller de la monarchie depuis son retour du premier voyage de Cook où le riche particulier s'était engagé comme botaniste, ou de James Edward Smith (1759-1828) qui se porte acquéreur, en 1783, des collections du Suédois Linné, collections qui formeront l'embryon des collections de la Linnean Society of London.

Comme très peu d'individus sont en mesure de réunir les capitaux nécessaires à l'achat de milliers de spécimens, les organisations étatiques émergent progressivement comme des acteurs majeurs des processus de centralisation des matériaux scientifiques. Derrière l'intégration verticale, se profile de plus en plus la main de l'État et la volonté des administrations de constituer des stocks de données nationaux à partir de la concentration de tous les dépôts pré-existants. Cette logique n'est nulle part mieux visible que dans l'entreprise révolutionnaire de constitution d'un herbier centralisé, réunissant des décennies d'accumulation d'échantillons au Jardin du Roi, dans ce qui est désormais le Muséum d'histoire naturelle, une institution ouverte à la nation. C'est ainsi qu'en 1793, sont fusionnées les collections distinctes de plusieurs botanistes, sous la houlette de René Louiche Desfontaines (1750-1833), à partir notamment du noyau de l'herbier de Vaillant, lui-même composite comme on l'a vu. L'intégration verticale des collections est l'échine dorsale du projet scientifique révolutionnaire, qui entend reconfigurer les anciens liens entre des objets éclatés pour les fondre dans le creuset d'une accumulation désormais ouverte à un public national. 
Mais, parallèlement à cette intégration verticale en cascade des spécimens, se développe un processus à la fois très différent et complémentaire : la prolifération horizontale des collections. Celle-ci est directement liée au premier processus. La formation de dépôts majeurs centralisés crée une incitation à la collecte pour des naturalistes engagés dans des relations de clientèle avec les savants métropolitains administrant ces collections verticales. Lors de leurs voyages, les naturalistes prélèvent ainsi des doubles des spécimens qu'ils échangent ensuite sur les marchés de l'information métropolitains ou qu'ils conservent pour leur propre compte. La pratique de la prise de « doubles » (échantillons multiples d'une même espèce) conduit à l'expansion horizontale des collections, truffées de copies, au dédoublement des objets collectés. Les doubles constituent le capital de travail des naturalistes en même temps que leur capital d'échange : le botaniste Ambroise Marie François Joseph Palisot de Beauvois(1752-1820) offre ainsi ses derniers spécimens de plantes africaines à son confrère britannique, l'influent botaniste Robert Brown (1773-1858), contre des spécimens convoités. Dans la lettre proposant l'échange, Palisot de Beauvois note qu'il a déjà largement distribué ses spécimens africains "à plusieurs botanistes en Europe ${ }^{9}$ ». Cette ubiquité des spécimens, dispersés en plusieurs exemplaires à l'échelle de tous les grands dépôts européens étatiques ou personnels, est incontrôlable. Même quand la collecte de spécimens s'effectue dans un cadre officiel et étatique (circumnavigations et voyages financés par les États), seule une partie des matériaux rejoignent les institutions d'État : les naturalistes conservent parfois pour leur propre usage des doubles qui se retrouvent ensuite échangés dans les réseaux scientifiques, démultipliant les ensembles collectés.

Les collections zoologiques issues des voyages de Cook comptent ainsi parmi les plus importantes collections extraeuropéennes des années 1770 : même si ces expéditions ont une double origine institutionnelle (Royal Society) et étatique (Amirauté) et que le projet de collecte vise ainsi à avancer les intérêts de la Couronne britannique dans les zones explorées, les objets ont ensuite été dispersées entre pas moins de cent dix-sept dépôts individuels ou publics, allant d'institutions dans plusieurs pays à des particuliers, qu'un article de Peter Whitehead répertorie dans le détail ${ }^{10}$.

Cette prolifération horizontale est renforcée par deux phénomènes. Le premier est la démultiplication des objets à partir des circumnavigations étatiques des années 1770 : l'intérêt étatique de plus en plus important et surtout organisé pour les matériaux extra-européens rencontre une demande croissante du public et des collectionneurs, le tout créant une incitation globale à la collecte et une augmentation mécanique du nombre de spécimens qui arrivent sur les réseaux d'échange scientifique européens. Le second phénomène est la percée, à la charnière des XVIII $^{\mathrm{e}}$ et XIX $^{\mathrm{e}}$ siècles, de collecteurs privés chassant des espèces rares pour leur propre compte ou celui de patrons scientifiques $^{11}$. Au début du XIx ${ }^{e}$ siècle, une société réunissant des collecteurs de plusieurs nationalités propose, sous le nom d'Unio itineraria, un système de souscriptions suivi d'une redistribution de spécimens collectés. Plusieurs col- 
lecteurs dont au moins un Français lui offrent leurs services. D'autres trouvent à s'employer au service de naturalistes institutionnels métropolitains et leur font parvenir des espèces nouvelles, moyennant rémunération. Ces collecteurs privés contribuent à la prolifération horizontale des spécimens disponibles, faisant cette fois l'objet de stratégies de commercialisation.

Parce que les pratiques scientifiques valorisent de plus en plus la nouveauté, accumulation verticale et dispersion horizontale sont étroitement liées. Seuls des dépôts massifs, centralisés ${ }^{12}$ semblent en mesure de permettre la comparaison des spécimens à la base de toute amélioration des taxonomies existantes; mais, dans le même temps, les scientifiques sont poussés à capitaliser sur les spécimens qu'ils possèdent et à les conserver pour eux comme capital de travail et de publication. Le même Desfontaines qui fusionne en 1793 les herbiers du Muséum d'histoire naturelle, écrit à son confrère anglais, le botaniste Robert Brown, en 1818, pour lui décrire les collections qu'attend le Muséum de l'activité de ses collecteurs officiels à Pondichéry, au Brésil, au Cap, à Madagascar; mais il ajoute ensuite qu'un autre voyageur nommé M. Pondeny vient d'aller à ses frais à la côte d'Afrique au-delà du Sénégal. "Celui-ci recueille des plantes pour moi particulièrement et s'il peut revenir sans accident je recevrai une collection que je partagerai avec vous $^{13}$. » La prolifération horizontale des matériaux se développe donc dans les interstices mêmes de l'accumulation verticale étatique.

Une autre formule révélatrice du botaniste français Auguste Duvau (1771-1831) souligne l'incitation à l'accumulation personnelle que crée le contexte de professionalisation savante au début du XIX ${ }^{\mathrm{e}}$ siècle. Écrivant au botaniste Nathaniel Wallich (1786-1854), du jardin botanique de Calcutta, en 1830, il ajoute : «Je ne crois pas avoir besoin de vous recommander les intérêts de ma propre collection ${ }^{14}$. » On pourrait multiplier les exemples, mais il apparaît clairement que les collections constituent un capital de recherche pour les naturalistes et qu'une forte incitation à conserver des ensembles de données distincts des collections nationales tire la dispersion horizontale des matériaux. Cette prolifération horizontale est plus importante et plus rapide que l'intégration verticale qui n'arrive pas à la compenser, comme en témoigne notamment le nombre élevé de doublons dans les identifications d'espèces au sein de la littérature savante. Les naturalistes travaillent de manière croissante, en matière de collections, dans un univers de la dispersion.

\section{Expansion de l'univers collectable}

La dispersion est donc le problème scientifique dont les contours se dessinent au $\mathrm{XVIII}^{\mathrm{e}}$ siècle. Non seulement la nature est dispersée au sens où les espèces de chaque embranchement sont réparties sur toute la surface du globe (chaque nouvelle découverte vient potentiellement modifier ou contredire 
les tentatives de trouver un ordre dans la nature), mais, en plus, les données qui la reflètent sont également dispersées.

L'éclatement des collections, aux mains d'une multitude d'acteurs privés, d'institutions nationales et de sociétés scientifiques différentes, représente un défi pour l'établissement de classifications fiables sur lesquelles se construit toute l'architecture technique d'analyse de la nature, alors que la taxonomie devient l'une des branches les plus cruciales des sciences naturelles au XVIII ${ }^{\mathrm{e}}$ siècle.

Comment surmonter l'expansion horizontale de l'univers collecté, représentant une masse de données toujours plus importante et toujours plus dispersée? Les problèmes de résolution des doublons reviennent constamment dans les correspondances en histoire naturelle, dont une partie du texte est fréquemment occupée par des tentatives de clarifier l'identité ou la différence d'objets différemment nommés. L'expansion de l'univers collectable se fait en partie en trompe-l'œil, alors que des erreurs d'identification ou des dénominations concurrentes multiplient artificiellement le nombre des espèces connues ${ }^{15}$, créant des surimpositions taxonomiques qui confèrent à cet univers de l'accumulation des matériaux une structure à plusieurs niveaux. Les réseaux académiques, à travers la normalisation des descriptions, des publications et des conditionnements d'objets, tentent depuis le xviII ${ }^{e}$ siècle d'enrayer cette entropie.

Outre le problème d'estimer quelle proportion de cet univers collectable est en fait composé de doublons, le second enjeu réside dans la logistique de l'accès à une infinité d'échantillons reposant entre les mains d'un nombre croissant de particuliers dans un grand nombre de pays, et de plus en plus dans les empires. La structure éclatée de cet univers de la collecte horizontale semble contredire les exigences croissantes de transparence motivant l'ouverture à des publics nationaux désireux d'accéder à ces stocks d'information.

Les revendications d'accès à ces collections privées se font plus insistantes dans les années 1770 et 1780 . L'un des champions de cette cause est le botaniste français Charles Louis L'Héritier de Brutelle (1746-1800). L'Héritier, riche particulier engagé dans des descriptions d'espèces de plantes nouvelles, partisan du système linnéen, est un personnage relativement excentré par rapport aux réseaux académiques français, auxquels il a substitué durant sa carrière des relations avec les botanistes d'outreManche. Conséquence directe de ses projets de publication d'espèces nouvelles, le travail de L'Héritier requiert une consultation récurrente des collections linnéennes. Dépendant de l'accès à des collections étendues pour son travail, disposant d'un accès réduit aux dépôts étatiques et en conflit avec des figures comme Jussieu ou Adanson, il n'est pas surprenant que L'Héritier ait développé tout un argumentaire en faveur $d^{\prime} u n$ droit d'accès aux collections privées. Le Français réclame plusieurs fois au fondateur de la Linnean Society et acquéreur des collections de feu Linné, l'Anglais James Edward Smith, l'accès à l'herbier du Prince de la botanique ${ }^{16}$.

L'expansion de cette nébuleuse de collections n'est donc pas qu'un phénomène mécanique, c'est aussi une lutte pour étendre les droits d'accès sur des spécimens-types souvent uniques, considérés 
par des naturalistes tels que L'Héritier comme relevant de la catégorie des biens communs, des choses publiques. De manière symptomatique, les individus comme L'Héritier se montrent aussi très critiques envers les difficultés d'accès aux dépôts étatiques où, selon eux, les objets pourrissent dans l'indifférence des savants académiques ${ }^{17}$, sur fond de mécontentement des jeunes botanistes linnéens contre l'intendance de Buffon au Jardin du Roi ${ }^{18}$.

Bien que clairement identifié par les naturalistes et collecteurs, le problème de l'expansion de l'univers collectable est structurellement insoluble à la fin du XVIII $^{\mathrm{e}}$ siècle parce que la tendance est à considérer que la valeur scientifique réside dans la masse des données. Une conception de plus en plus holistique des masses de matériaux porte l'accumulation tant étatique que privée, avec à la clef l'espoir de déchiffrer la structure du monde naturel. À la fin du XVIII ${ }^{\mathrm{e}}$ siècle, l'accumulation est érigée en système : il faut tout conserver, car tout est potentiellement important. Le patron de la Linnean Society, James Edward Smith, a cette formule méconnue mais extrêmement révélatrice de l'état d'esprit qui préside à l'accumulation de données : " The slightest piece of information which may tend to the advancement of the science we should thank fully receive. However trifling in itself, yet combined with other facts, it may become important ${ }^{19}$. "

Cette accumulation érigée en principe n'est pas du goût de tout le monde dans la communauté scientifique et fait l'objet de critiques théoriques. L'un des textes qui présente le mieux le sentiment des contemporains d'avoir affaire à une explosion informationnelle, à une dilatation des ensembles de données et d'objets et, par conséquent, à une expansion de l'univers collectable, est aussi une réfutation en règle de l'intérêt de collecter de grandes masses de données, inexploitables même lorsqu'elles sont organisées. L'article, publié dans le Journal de Physique, attaque les fondations mêmes du projet de collecte d'une information étendue :

" Une des grandes objections qu'on ne cesse de me faire, c'est que nos connoissances ou nos observations sont encore trop bornées, pour qu'on puisse ou qu'on doive y asseoir un système; qu'il faut donc s'en tenir à grossir la masse des faits particuliers, comme matériaux devant servir un jour à élever l'édifice; mais je vois qu'ils ne serviront jamais qu'à encombrer la place sur laquelle ils pourrissent. [...] Une observation géologique, quelconque ne pourrait jamais être utile qu'à celui-là seul qui l'a faite. [...] Il ne pourra jamais nous les transmettre. [...] Grand et triste exemple des peines et du Temps que nous perdons, pour ne laisser à nos neveux que des collections immenses de minéraux, descriptions, méthodes, dictionnaires ${ }^{20}$ ! »

L'accumulation a donc aussi ses mécontents, à l'heure où l'expansion de l'univers collectable crée un cadre désorientant qui rebat les cartes professionnelles. 


\section{Collections en réseau}

Parce que l'éclatement des objets amassés représente un nouvel enjeu pour le traitement des données, la manière dont est pensée la collection en sciences naturelles change aussi. Les liens qui peuvent être établis entre les données, par exemple entre divers spécimens, comptent autant sinon plus que les données et les spécimens eux-mêmes, dans le cadre de stratégies de recoupement et de mise en série. La circulation entre les objets accumulés devient partie intégrante de l'intérêt de la collecte.

Cette circulation peut consister en un déplacement des naturalistes pour examiner des stocks de matériaux conservés ailleurs. Le déplacement physique, par exemple, pour aller voir les collections de Joseph Banks ou de James Edward Smith à Londres (parmi les plus importantes collections privées du XVIII ${ }^{\mathrm{e}}$ siècle) est une pratique courante pour les botanistes français. Mais le déplacement géographique est une pratique inefficace quand des recherches imposent de comparer potentiellement des milliers de spécimens sans pouvoir les emporter pour référence ultérieure.

Tous les éléments connus, même dispersés, sont en effet nécessaires. La conception holistique des données mentionnée plus haut fait que même les trois ou quatre échantillons présents dans les vitrines d'un particulier peuvent être potentiellement utiles. Les sciences naturelles sont fortement dépendantes de spécimens uniques qui à eux seuls peuvent conduire à créer ou à supprimer des embranchements dans les classifications $^{21}$. C'est donc la somme de tous ces échantillons dispersés, mis en rela- tion par la somme de toutes les interactions scientifiques pour y accéder, qui constitue désormais la superficie de la collection. La collection, dans les sciences naturelles, englobe en puissance tous les matériaux connus et inventoriés puisqu'ils ont tous le potentiel de modifier les architectures classificatoires jusqu'ici admises. Par conséquent, la collection s'étend aussi loin que la capacité $\mathrm{du}$ naturaliste d'accéder aux matériaux nécessaires à son travail.

Le terme de collection vient à désigner, à la fin du XviII ${ }^{\mathrm{e}}$ et au début du XIX $x^{\mathrm{e}}$ siècle, un groupe de spécimens ou plus largement de données jointes à l'envoi d'une lettre : une série. Dans une lettre au botaniste Robert Brown, de 1814, son confrère français Palisot de Beauvois écrit qu'il regrette de ne pouvoir joindre " une collection plus considérable de [s]es plantes d'Afrique, j'en ai déjà tant donné à des botanistes de Paris et à plusieurs étrangers qu'il est des espèces dont il ne me reste plus qu'un seul échantillon, mais je vous en destine un de toutes celles que j'ai encore en double ${ }^{22} »$. Le terme de collection désigne un sous-ensemble de la masse connue des spécimens mis en circulation dans les réseaux scientifiques. Le botaniste Aylmer Bourke Lambert (1761-1842), membre fondateur de la Linnean Society et propriétaire d'une collection de cinquante mille spécimens incluant des espèces collectées par plusieurs naturalistes à bord des voyages de circumnavigation, écrit au naturaliste Charles Lucien Bonaparte (1803-1857), neveu d'un Bonaparte plus connu et ornithologue réputé, qu'il « lui a offert 
une large collection de plantes nouvelles, $\mathrm{d}^{\prime}$ Inde et d'ailleurs ${ }^{23}{ }^{\prime}$.

Dans ces occurrences, la collection désigne le transfert de données. La collection devient donc une série - notion très importante dans les sciences naturelles - sélectionnée pour la communication. Elle est formatée par son caractère circulatoire. Le graphe qui suit (fig. 19, cahier couleur), tiré de nos travaux doctoraux ${ }^{24}$, cartographie les échanges de données et d'échantillons entre naturalistes britanniques et français dans leurs correspondances sur un long XVIII ${ }^{\mathrm{e}}$ siècle. La collection correspond virtuellement au graphe : c'est l'ensemble des objets accessibles, avec une proximité variable suivant les individus et leur centralité dans cette toile d'interactions.
On est ici en présence de ce qui constitue l'un des premiers réseaux globaux de traitement de données, qui n'est pas structuré mais émergent. L'articulation de ces collections distribuées en réseau se fait par des milliers de transferts ponctuels de données entre correspondants dans les sciences naturelles, relayés par une presse de plus en plus rapide et globalisée.

Les réseaux d'échange scientifiques deviennent un dispositif technique essentiel à l'exploitation des stocks de matériaux et d'échantillons disponibles : une infrastructure décentralisée et évolutive, adaptée à une collection globale en perpétuelle mutation.

\section{Mutation dans I'architecture technique des savoirs}

Ce caractère réticulaire qui émerge dans la collection en sciences naturelles à mesure que la discipline implique la gestion de masses de plus en plus importantes de spécimens, entraîne des mutations dans l'architecture technique des savoirs et dans les pratiques scientifiques.

Une première conséquence en dérive : il est relativement inutile de posséder soi-même tous les échantillons accessibles via le réseau. Ce qui importe est de conserver une capacité d'accès à ces objets, ménagée via des contacts et des intermédiaires. On peut parler d'externalisation des données et des spécimens : il n'est pas nécessaire de tout posséder ou centraliser, il suffit de localiser les objets dans le réseau puis d'en obtenir une copie ${ }^{25}$ (dessin, description ou encore mieux l'original). Le titulaire de la chaire d'histoire naturelle de l'université de Montpellier, Michel Félix Dunal (1789-1856), écrit à Robert Brown, en 1816, pour lui présenter son premier ouvrage, la monographie des Solanum. En vue de la seconde édition, il cherche à rassembler

" les matériaux qui peuvent se trouver sur ce sujet dans les collections que je n'ai pu voir, et cela pour rendre ma seconde édition plus exacte et plus complète. Vous possédez plusieurs espèces de Solanum de la Nouvelle-Hollande qui ne sont signalées que par des phrases dans votre admirable Prodromus. 
Sur 19 espèces qui y sont indiquées 14 me sont inconnues. À la vérité, j'ai cru reconnaître les Solamumbiflorum et violaceum dans l'herbier du Muséum de Paris mais je ne suis pas certain de ne pas avoir fait des erreurs. [...] Il est bien indiscret de ma part de vous demander la communication directe ou par l'intermédiaire de M. de Candolle, des espèces que vous possédez [...] ou si vous l'aimiez mieux la communication de vos descriptions ${ }^{26}{ }^{»}$.

La collecte, dans la collection, devient davantage une question de localisation des spécimens dans l'écosystème des communautés scientifiques que d'acquisition par soi-même de grandes masses d'échantillons sur le terrain. Cette redirection de l'effort vers la localisation des données utiles au sein d'un écosystème informationnel éclaté ne place pas tous les naturalistes sur un pied d'égalité. Ceux qui tendent à se situer au centre du graphe (c'est-à-dire les mieux connectés), souvent des naturalistes métropolitains disposant de nombreux sous-réseaux d'informateurs et occupant une place nodale dans les réseaux institutionnels, ont à la fois une meilleure visibilité des ressources disponibles mais sont, en même temps, beaucoup plus saturés d'information. En multipliant les intervenants, la collection en réseau a le potentiel de générer du « bruit » (défini comme des données redondantes ou une absence d'information), tout autant que d'en supprimer. Doublons et erreurs accompagnent inévitablement la redirection de l'infrastructure informationnelle vers une dimension planétaire et connectée. L'efficacité de l'organisation technique des savoirs dépend dès lors de l'architecture du réseau, ouvrant des perspectives de recherches faisant appel à l'analyse de graphes ${ }^{27}$. Le point capital est que la collecte est prise dans des relations d'interdépendance : l'accumulation d'objets naturels si nécessaire aux sciences de la vie ne peut plus être comprise comme l'effort d'une institution centrale ou d'un riche particulier, c'est la résultante des forces de collecte dans tout le réseau, réseau qui forme l'étendue potentielle maximale de la collection.

Une deuxième conséquence en découle directement : la force de traitement informationnelle réside maintenant dans les potentialités de connexion des données, à l'intersection des dépôts d'objets. Pour tirer sens des ensembles de données, il faut les mettre en relation. Recoupements et comparaisons des données sont une fonction de la circulation des matériaux. Alors que les États plaçaient leur capacité de traitement des données scientifiques dans l'accumulation au sein de grands dépôts centralisés nationaux, le réseau offre une force de traitement distribuée. La circulation des données offre une alternative à leur concentration comme moyen de résoudre des problèmes scientifiques : là où les dépôts étatiques misent sur la concentration localisée des matériaux, la collection éclatée mise sur leur connexion à grande échelle. La collection en réseau réalise à plus grande échelle, et sans dépendre du paramètre de la distance, ce que les dépôts étatiques tentent dans le périmètre limité d'institutions choisies. De ce point de vue, l'ensemble d'un graphe est potentiellement plus performant que les collections étatiques même les plus complètes et centralisées; 
notamment s'il existe au moins un point d'accès aux réseaux étatiques de collecte en son sein. L'un des naturalistes les plus importants de la période, le Britannique Joseph Banks (1743-1820), en est un bon exemple qui met en mouvement autour de lui un réseau étendu d'informateurs, tout en détenant un accès privilégié aux collections institutionnelles (jardins de Kew, Royal Society). La jonction entre la capacité d'expertise qu'offrent les collections académiques concentrées et la force de traitement distribuée qu'offrent des réseaux à large portée est certainement l'une des structures les plus payantes. Mais, sur le long terme, à mesure que l'accès aux spécimens des zones extraeuropéennes pèse plus lourd dans les logiques de publication, l'étendue du réseau, cette force de traitement de la collection éclatée, devient plus déterminant.

Et c'est là la troisième conséquence. Dans la pratique technique des naturalistes, cet éclatement (le fait que les ensembles d'objets ne sont jamais réunis dans leur entièreté au même endroit) a pu limiter les incitations à un traitement monographique et sectoriel des collections et encourager au contraire une recherche à l'échelle globale portant, par exemple, sur la distribution de certaines espèces. Au fur et à mesure que le XVIII $^{\mathrm{e}}$ siècle avance, les espèces et les familles non décrites à portée des naturalistes en Europe se raréfient. Pour percer sur le marché de la publication scientifique, porté par une presse en plein essor, il faut chercher plus loin des espèces inconnues, ce qui nécessite des comparaisons avec celles déjà décrites ; ou bien il faut traiter de groupes d'êtres vivants qui sont eux-mêmes dispersés, par exemple les Solanum recherchés par Dunal plus haut (1500 espèces sur plusieurs continents). Un réseau à portée globale acquiert un avantage décisif dans ce nouveau paysage des pratiques scientifiques. La collection en réseau, de ce point de vue, a pu encourager une heuristique globale des espèces, par exemple des différences de distribution $^{28}$. Le naturaliste et explorateur Alexander von Humboldt (1769-1859), en quantifiant des données portant sur des espèces de diverses régions, est l'un des initiateurs du tournant biogéographique dans les sciences naturelles à la fin du XvIII $^{\mathrm{e}}$ siècle. Ce saut d'échelle, permis par une mutation dans l'accès aux matériaux pensés en réseau, est très important pour la naissance de disciplines comme la biogéographie qui sont lourdement dépendantes d'une collecte globale des espèces, collecte impossible si elle n'est pas largement distribuée ${ }^{29}$.

\section{Protocoles de formalisation}

Si la nature émergente ${ }^{30}$ et non codifiée de ce réseau d'échantillons et de données est précisément la condition qui lui permet d'acquérir une masse critique supérieure à celle de n'importe quel dépôt institutionnel, il existe néanmoins des tentatives d'organisation des liens entre les contenus. Au XVIII ${ }^{\mathrm{e}}$ siècle, les instructions aux voyageurs ${ }^{31}$ émanant des académies ou de l'échelon éta- 
tique encouragent la standardisation de l'observation et la formalisation du langage de description. Néanmoins, si les instructions de voyage simplifient le formatage des données, elles ne prévoient pas de dispositif pour les comparer entre elles et n'opèrent pas directement dans cet espace d'intersection entre les collections que nous décrivons ici (ou, si l'on préfère, au niveau de la connexion des données). Au début du Xix ${ }^{e}$ siècle, quelques naturalistes commencent à cerner les contours de cet espace d'intersection, en réfléchissant à une formalisation des structures d'échanges liant les stocks de données disponibles. Le botaniste William Jackson Hooker (17851865) esquisse ainsi les linéaments d'un système de comparaison systématique des collections privées et publiques, visant à réduire le nombre des doublons dans la littérature scientifique :

"Sans comparaison des plus larges collections les unes avec les autres, par exemple de celle de Berlin avec celle de Paris, et de l'une ou l'autre avec les herbiers de Banks ou Lambert, on ne peut s'attendre à aucun degré de certitude; et, en raison de l'accroissement de collections privées extensives et non vérifiées, la science doit travailler avec le lourd désavantage de l'accumulation des synonymes qui en résultent. Si Sieber avait identifié ses plantes collectées en Crète et en Égypte avec les nombreuses autres précédemment rapportées par Sibthorpe et Desfontaines, de nombreux doutes auraient disparu; si les récents voyageurs au Brésil, le Prince Nieuwied, Auguste St. Hilaire, Mar- tius et Pohl, avaient comparé leurs trésors avant de les décrire, bien des synonymes [doublons] inutiles n'auraient jamais existé. Voyager d'un herbier à l'autre en emportant dans sa seule mémoire les caractères $\mathrm{d}$ 'espèces douteuses est assimilable à une tâche impossible, et la confusion que crée de telles entreprises est visible dans l'un de nos plus importants ouvrages botaniques ${ }^{32}$. »

À la même époque, des tentatives similaires de mise en réseau formelles sont expérimentées par le baron d'Audebert de Férussac (1786-1836), qui pose les bases du premier système global de partage de nouvelles scientifiques avant Internet, via la presse. Son Bulletin général universel, prototype de veille scientifique agglomérant des données globales, paraîtra entre 1823 et $1830^{33}$. Le géologue autrichien Ami Boué (17941881) expérimente lui aussi avec une organisation plus formelle, en ébauchant un système de mise en réseau des collections de périodiques scientifiques, insuffisamment disponibles selon lui dans les bibliothèques parisiennes ${ }^{34}$.

C'est ainsi qu'entre 1800 et 1830, une réflexion émerge sur l'utilisation des réseaux comme infrastructure technique d'articulation et d'analyse de collections désormais globales dans leur composition biologique comme dans leur localisation géographique. La collection scientifique pensée comme un ensemble de processus de circulation des données ouvre la voie aux réflexions contemporaines sur les pratiques d'externalisation ouverte (crowdsourcing ${ }^{35}$ ) et de mise en réseau des collections. 
C'est aussi au tournant du siècle qu'émerge un questionnement sur le langage formel qui permet la communication des données et échantillons scientifiques à l'échelle de réseaux étendus, ce qu'on peut qualifier de protocoles de formalisation, notamment parce que des naturalistes commencent à réfléchir à la nécessité d'une standardisation du langage. Le botaniste et collecteur Jacques Julien Houtou de la Billardière (17551834), à la poursuite de ses collections capturées sur un vaisseau hollandais pris par les Anglais au Cap, écrit à James Edward Smith, en 1796 :

«Faites à ce sujet, mon ami, toutes les recherches possibles. Vous savez combien il y aurait à perdre pour la science si des collections de cette nature n'étaient pas remises entre les mains de celui qui les a faites, les observations étant transmises au moyen d'abbréviations qui ne sont connues que de lui et qui lui rappellent une infinité de choses confiées en partie à sa mémoire. »

Sans standardisation, la communication des notes et des objets est pratiquement inutile. La Billardière esquisse la dernière dimension manquante à une collection globale véritablement fonctionnelle : un langage formel, la structurant de manière identique dans tout le réseau.

$\mathrm{Au}$ tournant du siècle, tous les concepts clef d'une collection en réseau ont émergé : la notion de séries, la force de traitement d'un réseau global, la reconnaissance de la capacité d'accès aux matériaux comme un élément déterminant des pratiques professionnelles scientifiques. Ne manque qu'un langage technique formel, une syntaxe, pour unifier cet univers de données en expansion. Sans un tel langage d'organisation des notes et échantillons, on limite la réutilisation des données hors des cercles qui les ont produites. Mais que l'on ajoute cette syntaxe, ce langage structurant, et l'on approche du prototype (ou de l'ancêtre) de la base de données, de la collection digitale ouverte qu'incarne, par exemple, un projet informatique en ligne comme Biodiversity Heritage Library, équipé d'un système de reconnaissance globale de noms d'espèces ${ }^{36}$. La collection digitale que nous connaissons, celle $\mathrm{du}$ Web, se dessine dans le prolongement logique de la collection virtuelle ici décrite : de l'intersection, elle fait une syntaxe.

\section{Conclusion}

Tandis que les espaces nationaux de conservation des spécimens et des données se structurent en centres principaux de l'accumulation de l'information à la fin $\mathrm{du} \mathrm{xvIII}^{\mathrm{e}}$ siècle, une construction parallèle, et gigantesque, se développe

dans les interstices de l'accumulation institutionnelle métropolitaine. Les masses de matériaux entre les mains de particuliers, cette collection éclatée, représentent de très loin le plus important gisement informationnel, devant l'accumulation 
étatique. La structure réticulaire de cet ensemble, en soi une technologie d'articulation des données, bouleverse les pratiques scientifiques et crée un puissant filtre en ce début du $\mathrm{xIX}^{\mathrm{e}}$ siècle, à l'heure de la professionnalisation dans les sciences naturelles, dissociant lentement les segments de la communauté scientifique qui parviennent à exploiter cette structure de ceux qui pointent les nouveaux problèmes que pose la dispersion. Si la collection est partout à la fois, ce sont les liens entre les stocks privés, ou privés et publics, qui créent l'unité de cette infrastructure globale : la collection scientifique structurée à l'échelle de réseaux étendus est avant tout un ensemble d'hyperliens dont la maîtrise conditionne les recoupements qu'il est possible d'effectuer au sein de l'information biologique existante et, au-delà, la direction que prennent les sciences naturelles dans leurs modèles du globe et des espèces.

\section{Notes}

1. Cette observation fondamentale est formulée dans Martin Rudwick, Bursting the limits of time : the reconstruction of geohistory in the Age of Revolution, Chicago, University of Chicago Press, 2007, p. 251 note 10

2. Par extension, l'analyse ou la mise en relation de ces données constitue ce que nous désignerons sous le nom d'information.

3. Nous empruntons l'expression de MarieNoëlle Bourguet, « La collecte du monde : voyage et histoire naturelle, fin $\mathrm{XVII}^{\mathrm{e}}$ siècle-début XIX ${ }^{\mathrm{e}}$ siècle ", in Claude BLANCKAERT, Claudine Cohen, Pietro Corsi, Jean-Louis Fischer (dir.), Le Muséum au premier siècle de son histoire, Paris, Muséum national d'histoire naturelle, 1997, p. 163-196.

4. Sarah IRving, Natural sciences and the origins of the British Empire, Empires in perspective number 5, Londres, Pickering \& Chatto, 2008. Sur le Repository de la Royal Society, Matthew C. Hunter, Wicked intelligence. Visual art and the science of experiment in Restoration London, Chicago, University of Chicago Press, 2013.

5. Recherche d'occurrences avec l'outil Ngram Viewer, qui exploite le plus vaste corpus d'imprimés numérisés et traités par reconnaissance de caractères exploitable à l'heure actuelle, celui de Google Livres. L'expérience est reproductible avec des graphes interactifs à l'adresse suivante : [https://books.google.com/ngrams]. Paramètres : entre 1700 et 1900, terme « collection », corpus en langue anglaise, smoothing $=3$. Google Ngram Viewer, 2017.

6. Sur les collections dans un cadre impérial, Bertrand DAUgERON, Collections naturalistes, entre science et empires, 1763-1804, Paris, Éditions du Muséum national d'histoire naturelle de Paris, 2009.

7. Royal Society, Sherard papers MS 252, Danty d'Isnard à Sherard, lettre 259, Paris, 11 avril 1722.

8. Les collections de Tournefort et de Vaillant en forment l'embryon. L'herbier de Vaillant a servi de noyau et a été fusionné avec la collection générale ; l'Herbier Tournefort, rangé selon sa méthode décrite, a été conservé intact, avec l'appellation d'Herbier historique; Denis LAMY et Aline Pelletier, « La conservation et la valorisation de l'Herbier de Tournefort au Muséum national d'histoire naturelle ", Lettre de l'OCIM, 130, 2010, p. 19-26.

9. British Library, Add. 32439, vol. I., New Holland : letters and papers of a scientific expedition to 1800-1806, f. 351, Ambroise Marie François 
Joseph de Palisot de Beauvois à Robert Brown, Paris, 3 mai 1812.

10. Peter James Palmer WhiteheAd, «A guide to the dispersal of zoological material from Captain Cook's Voyages », Pacific Studies, 2, 1, 1978, p. 52-93.

11. Dont les Français employés par William Jackson Hooker autour de l'Océan indien, exemple : Library and Archives at Royal Botanic Gardens, Kew Relation Directors'Correspondence 52/6L, [Louis] Bouton à Sir William Jackson Hooker, Maurice, 13 septembre 1830.

12. Sur le rôle du moment révolutionnaire en France dans la constitution de tels dépôts et d'un processus de concentration, on consultera avec profit Pierre-Yves Lacour, La République naturaliste : collections d'histoire naturelle et Révolution française (1789-1804), Paris, Publications scientifiques du Muséum national d'histoire naturelle, 2014.

13. British Library, Add. 32440, vol. II., 18131825, Desfontaines à Brown, Paris, 26 avril 1818, f. 194.

14. BCMHN, ms CRY $502 / \mathrm{n}^{\circ} 527$, Auguste Duvau à Nathaniel Wallich, Paris, 10 avril 1830.

15. Jim Endersby a montré que l'inflation du nombre des espèces avait une logique géographique : les collecteurs ont tendance à présenter comme nouvelles des espèces sur lesquelles ils construisent leur carrière scientifique à la périphérie, tandis que, dans les centres métropolitains, les savants sédentaires procèdent à une réduction du nombre des espèces comme une démonstration de contrôle informationnel sur lequel repose leur centralité. Jim ENDERSBY, Imperial nature : Joseph Hooker and the practices of Victorian science, Chicago, University of Chicago Press, 2008.

16. La correspondance entre L'Héritier et James Edward Smith conservée à la Linnean Society insiste de manière récurrente sur la nécessité de cette ouverture; Linnean Society, Smith Papers 5.91, L'Héritier à Smith, Paris, 14 mars 1785; 5.95 L'Héritier à Smith, Paris, 20 avril 1785.

17. British Library, Add. MS.8095 188-189, L'Héritier à Banks, Paris, 2 février 1783, 293-294 ; L'Héritier à Banks, Paris, 7 juin 1783.

18. Pascal Duris, Linné et la France, 1780-1850, Genève, Droz, 1993.

19. James Edward SMITH, « Introductory Discourse on the rise and progress of Natural History ", 8 avril 1788, Transactions of the Linnean Society, 1 (1791), p. 52 : «Nous deviendrons recevoir avec gratitude la moindre information susceptible de tendre à l'avancée de la science. Si insignifiante en elle-même soit-elle, combinée avec d'autres éléments, elle peut se révéler importante. »
20. Philippe M. Bertrand, « Mémoire sur les questions élémentaires ou fondamentales d'une Théorie de la Terre ", Journal de physique, de chimie, d'histoire naturelle et des arts, 49 (1799), p. 121.

21. Voir note 1.

22. British Library, Add. 32440, vol. II., 18131825, f. 58, Palisot à Brown, Paris, 15 octobre 1814.

23. BCMHN, ms 2605, $n^{\circ} 1853$, Aylmer Bourke Lambert à Bonaparte, Londres, 21 juin 1827.

24. Thérèse BRU, Circulations scientifiques. Les naturalistes et leurs données entre les mondes britanniques et français (1700-1836), thèse de doctorat d'histoire sous la dir. de Philippe Minard, 2017.

25. Sur les dispositifs de copie et de transposition de l'information, notamment la transition entre les trois dimensions de l'objet et les deux dimensions de la reproduction visuelle, Thérèse BRU, « Plus vrai que nature. Conversion des objets en information scientifique, conversion de l'information en objets dans les correspondances en sciences naturelles ", in Thérèse BRU et Solène de La Forest D'Armaillé, Matière à écrire. Les échanges de correspondance $d u \mathrm{XVI}^{e}$ au XIX ${ }^{e}$ siècles, Vincennes, Presses universitaires de Vincennes, coll. Temps et espaces, 2017, p. 179-203.

26. British Library, Add. 32440, vol. II, 18131825, f. 121, Michel Félix Dunal à Brown, Montpellier, 15 septembre 1816.

27. Dimension centrale de nos travaux doctoraux, voir note 24 . Il s'agit d'un problème d'optimisation de la circulation de données, influencé par la manière dont se structure le graphe.

28. Janet BROWNE, The secular ark: studies in the history of biogeography, New Haven, Yale University Press, 1983.

29. Et au-delà, de théories scientifiques comme celle de Darwin, si on considère la part que tient dans ses écrits la corrélation de données venant du monde entier.

30. Constituée par un ensemble d'interactions dont les effets sont supérieurs à la somme.

31. Joan-PauRubiÉs, «Instruction for travellers : teaching the eye to see ", History and Anthropology, $\mathrm{n}^{\circ}$ 9, 1996, p. 139-190; Antonella VANNONI, « Les instructions pour les voyageurs : voyage, expérience et connaissance au XVIII ${ }^{\mathrm{e}}$ siècle ", in Claude BLANCKAERT (dir.), Le terrain des sciences humaines (XVIII ${ }^{e}-X^{e}$ siècles), Paris, L'Harmattan, 1996, p. 73-88; Silvia Collini, «Conseils pratiques et orientations théoriques dans les instructions pour les voyageurs (xvIII siècle) », ibid., p. 57-72; Lorelai KURY, « Les instructions de voyage dans les expéditions scientifiques françaises (17501830) ", Revue d'histoire des sciences, $\mathrm{n}^{\circ}$ 51-1, 1998, p. 65-92; Silvia Collini, Antonella VANNONI (dir.), 
Les instructions scientifiques pour les voyageurs, XVII XIX ${ }^{e}$ siècle, Paris, L'Harmattan, 2003.

32. William Jackson HOоKeR, Botanical miscellany : containing figures and descriptions of such plants as recommend themselves by their novelty, rarity or history, vol. 1, 1830, p. 51-52.

33. Bernard BRU, Thierry MARTIN, " Le baron de Férussac, la couleur de la statistique et la topologie des sciences ", Journal électronique d'histoire des probabilités et de la statistique, 1, 2, 2005, p. 1-43.

34. BCMHN, ms 2533 / n 16 Ami Boué, «Sur la nécessité d'avoir à Paris un local ou établissement public contenant tous les journaux scientifiques et les recueils des sociétés savantes publiés dans le monde entier ", slnd.

35. Voir le projet Constructing Scientific Communities (Université d'Oxford), sous la dir. de Sally Shuttleworth et Chris Lintott, investiguant le rôle de l'externalisation ouverte dans les sciences depuis le XIX siècle, dans un contexte où l'information à traiter devient exponentielle et n'est plus gérable par un cercle restreint de scientifiques. https: [//conscicom.org/].

36. Consortium de bibliothèques visant à numériser les collections relatives à la biodiversité et aux sciences naturelles http: [//www.biodiversitylibrary.org/]. 